\title{
Servo Device
}

National Cancer Institute

\section{Source}

National Cancer Institute. Servo Device. NCI Thesaurus. Code C50169.

An electronic device designed to amplify the force of a movement. 